\title{
Paracrine effect of human adipose-derived stem cells on lymphatic endothelial cells
}

\author{
Cristiana Marcozzi ${ }^{(\mathbb{D})}$, Annalisa Frattini², (D), Marina Borgese ${ }^{4}$, Federica Rossi ${ }^{4}$ (D), Ludovica \\ Barone $^{4}$ (D), Eleonora Solari ${ }^{1}$ (D), Roberto Valli ${ }^{3}$ \& Rosalba Gornati ${ }^{* 4}$ (iD \\ ${ }^{1}$ Department of Medicine \& Surgery, Human Physiology, University of Insubria, 21100 Varese, Italy \\ ${ }^{2}$ Institute for Genetic \& Biomedical Research, CNR, 20138 Milano, Italy \\ ${ }^{3}$ Department of Medicine \& Surgery, Human and Medical Genetics, University of Insubria, 21100 Varese, Italy \\ ${ }^{4}$ Department of Biotechnology \& Life Sciences, University of Insubria, 21100 Varese, Italy \\ *Author for correspondence: Tel.: +39 033242 1314; rosalba.gornati@uninsubria.it
}

Aim: The proposal of this study was to evaluate, in vitro, the potential paracrine effect of human adiposederived stem cells (hASCs) to promote lymphangiogenesis in lymphatic endothelial cells isolated from rat diaphragmatic lymphatic vessels. Materials \& methods: ELISA on VEGFA, VEGFC and IL6 in hASCconditioned medium; LYVE1 immunostaining; and gene expression of PROX1, VEGFR3, VEGFC, VEGFA and IL6 were the methods used. Results: In 2D culture, hASC-conditioned medium was able to promote lymphatic endothelial cell survival, maintenance of endothelial cobblestone morphology and induction to form a vessel-like structure. Conclusion: The authors' results represent in vitro evidence of the paracrine effect of hASCs on lymphatic endothelial cells, suggesting the possible role of hASC-conditioned medium in developing new therapeutic approaches for lymphatic system-related dysfunction such as secondary lymphedema.

Lay abstract: This work is addressed to provide, by scientific experiments, information about the possibility of correcting localized swelling of the body caused by an abnormal accumulation of lymph using culture medium enriched with metabolites, growth factors and extracellular matrix proteins secreted by a stem cell population. The authors' results could be an innovative therapeutic approach for regenerative medicine and may have an important impact on the treatment of lymphatic-related pathologies.

First draft submitted: 18 May 2020; Accepted for publication: 19 October 2020; Published online: 17 November 2020

Keywords: conditioned medium $\bullet$ ELISA $\bullet$ gene expression $\bullet$ immunohistochemistry $\bullet$ lymphangiogenesis

Human adipose-derived stem cells (hASCs) are mesenchymal stem cells that are isolated from adult adipose tissue, accessible in large quantities with minimal invasive harvesting procedures and efficiently grown in vitro [1,2]. These hASCs, because of their self-renewal, multi-lineage differentiation capacity $[3,4]$ and ability to release biologically active molecules [5-7], are promising as a new therapeutic approach for stem cell-based therapies.

Increasing evidence suggests that stem cells mediate tissue repair through the release, into the site of the lesion, of factors that can activate, by paracrine effect, specific molecular pathways involved in tissue repair [8]. This hypothesis is supported by in vitro and in vivo studies showing that many cell types respond to paracrine signaling by modulating cellular responses, such as survival, proliferation, migration and gene expression. In particular, hASC transplantation promotes angiogenesis and tissue regeneration, inhibiting processes like fibrosis, apoptosis and inflammation $[9,10]$.

There is evidence that hASCs secrete several pro-angiogenic factors, such as HGF, VEGFs, PDGF, FGFs, IL6, Ang 1 and 2 and anti-apoptotic and anti-inflammatory factors [5,11-15]. These bioactive factors may be easily collected in the hASC culture medium (conditioned medium $[\mathrm{CM}]$ ), which has been reported to have similar effects, in terms of tissue repair processes, as those exerted by the cells when inoculated into animal models [16,17]. The paracrine effect of hASCs on angiogenesis and revascularization of ischemic tissue is well documented [18,19], and recent data suggest their implication in lymphangiogenesis [20]. Future
Medicine 
The lymphatic system plays a critical role in maintaining tissue fluid homeostasis, lipid absorption and immune cell trafficking. Extrinsic and intrinsic mechanisms sustain lymph drainage and transport along the lymphatic network. The extrinsic process depends upon the transmission of mechanical forces arising in tissues surrounding lymphatic vessels [21-23], whereas the intrinsic process relies on rhythmic, spontaneous contractions of lymphatic muscle cells in the vessel wall itself [24-26].

Dysfunction in lymphatic vessels leads to impaired drainage, resulting in the development of lymphatic-related diseases such as lymphedema, a pathological condition characterized by localized fluid retention and tissue swelling and associated with the risk of recurrent infections, ulcers, fibrosis, lipid accumulation and metastasis of malignant diseases [27]. Primary lymphedema is a genetic disease that is caused by congenital conditions such as Milroy disease and leads to incomplete or abnormal vessel formation [28-30]. By contrast, secondary lymphedema is commonly the result of complications after lymph node dissection (i.e., surgical sentinel lymph node removal) combined with radiation therapy in cancer treatment [31]. Secondary lymphedema mainly affects the upper and lower limbs and causes disfiguring conditions and pain, with a significant social impact on a patient's lifestyle. Current applicable therapies involve bandaging, massage and compressive techniques [32], which act as extrinsic pumps, increasing local tissue pressure; however, these are transient solutions that alleviate a patient's symptoms but do not allow for the recovery of physiological lymph flow and, in general, lymphatic function. From this perspective, recent research conducted in animals [33] as well as in a human pilot study [34] has reported the effect of hASC transplantation, highlighting the regenerative pro-lymphangiogenic effect.

Considerable progress has been made in the identification of the molecular mechanisms that regulate lymphangiogenesis, although some of the signaling pathways still need to be clarified. The first powerful pro-lymphangiogenic factor that was discovered was VEGFC, which, by binding to VEGFR3 [35-37], plays a pivotal role in promoting lymphatic endothelial cell (LEC) survival and organization into functional lymphatic vessels [38,39]. Moreover, VEGFC, through the activation of VEGFR3, improves lymphedema-inducing endothelial cell proliferation and migration, inhibits apoptosis and restores lymph flow by ameliorating the lymphatic pump activity of the collecting vessels [20,40-42]. The role played by VEGFR3 in lymphatic system development and physiological function has been demonstrated in animal models [43] and is supported by evidence that, in humans, mutation of the VEGFR3 gene induces primary lymphedema (LMPH1A, OMIM 153100) [44,45]. Moreover, a recent study has shown that VEGFR3 is implicated in the regulation of spontaneous lymphatic contractility, highlighting its pivotal role as a potential pharmacological target [46].

Recent works, reporting the paracrine effect of hASCs on LECs, represent a good starting point for investigating the role of the hASC paracrine effect on lymphangiogenesis $[41,42,47-49]$. From this perspective, the aim of the current study was to evaluate the paracrine effect of CM, derived from cultured hASCs (hASC-CM), on primary LEC culture. The authors' main interest was to assess whether any beneficial effect exerted on cultured LECs could be due to hASC-CM as a combination of complementary growth factors.

The hASCs were obtained from the adipose tissue of healthy subjects, and primary LEC culture was derived from rat diaphragmatic lymphatic networks. In this work, instead of using LECs derived from human dermal lymphatic vessels, LECs were isolated from microlymphatic vessels obtained from rat muscular diaphragmatic specimens. This choice was related to the tissue of origin, which is strictly connected to the surrounding interstitium and deeply affected by its mechanical features, which are different from those of the dermis $[21,23,50]$.

The authors' data showed that hASC-CM was able to promote LEC survival and maintenance. Furthermore, the current evidence supports the concept that hASC-CM may potentially be used to treat, improve or prevent lymphedema.

\section{Methods}

\section{Samples}

All subjects gave their informed consent to be included in this study, and all procedures were performed in accordance with the Ospedale di Circolo Ethical Committee and the European Communities Council Directive of EU/63/2010. The Italian Ministry of Health (192/2013) approved the experimental study.

Human adipose tissue was obtained by surgical intervention on healthy young women $(\mathrm{n}=3$; age: $33.4 \pm 5.3$ years) who underwent breast reduction because of gigantomastia. All subjects were nonsmokers, had not experienced any great weight loss from dieting (BMI: $25.6 \pm 2.6 \mathrm{~kg} / \mathrm{m}^{2}$ ), had no history of metabolic disorders and were not taking medications at the time of the medical procedure. 


\section{hASC isolation \& culture}

The hASCs were isolated from human mammary adipose tissue according to Gronthos and Zannettino [51] following a modified protocol [52]. Briefly, the stromal vascular fraction was obtained after collagenase type II digestion $\left(3 \mathrm{mg} / \mathrm{g}\right.$ tissue; Sigma-Aldrich, Milan, Italy) at $37^{\circ} \mathrm{C}$ for $1 \mathrm{~h}$ in agitation. After filtration $(100-\mu \mathrm{m}$ cell strainers), the solution was centrifuged at 180 relative centrifugal force (RCF) for $10 \mathrm{~min}$ at room temperature (RT). The resulting pellet was washed with erythrocyte lysing buffer $\left(154 \mathrm{mM} \mathrm{NH}_{4} \mathrm{Cl}, 10 \mathrm{mM} \mathrm{KHCO} 3\right.$ and $1 \mathrm{mM}$ EDTA). Cells were then seeded in T25 flasks in complete DMEM:DMEM/nutrient mixture F12 at 1:1 (Sigma-Aldrich) supplemented with $2 \mathrm{mM} \mathrm{L-glutamine,} \%$ penicillin-streptomycin, $0.1 \%$ gentamicin and $10 \%$ fetal bovine serum (FBS) and incubated at $37^{\circ} \mathrm{C}$ and $5 \% \mathrm{CO}_{2}$. After $6 \mathrm{~h}$, culture medium was replaced with fresh medium to remove non-adherent cells. The hASCs were subsequently cultured in T75 flasks and used at the fifth passage, at $70-80 \%$ confluence, for all experiments.

\section{Cytogenetic analysis of hASCs}

To define genome stability, hASC cytogenetic analysis was performed at passages two, four, ten and 12. Chromosomes from hASCs were prepared by standard cytogenetic protocol. Briefly, hASCs were cultured in T25 flasks in $5 \mathrm{ml}$ of DMEM supplemented with 10\% FBS and 1\% penicillin-streptomycin. Cells were then treated for $3 \mathrm{~h}$ with $20 \mu \mathrm{l}$ of $10 \mu \mathrm{g} / \mathrm{ml}$ colcemid solution (Euroclone, Milan, Italy), detached by trypsin/EDTA treatment, diluted in DMEM plus $10 \%$ FBS to block protease digestion and centrifuged at 200 RCF for 5 min at RT. Supernatant was removed and pellet suspended dropwise in $\mathrm{KCl} 56 \%$ hypotonic solution for $15 \mathrm{~min}$. The hASCs were recovered by centrifugation and resuspended gently dropwise in standard methanol/acetic acid 3:1 fixative solution. The fixing process was repeated twice, then cells were incubated at $4^{\circ} \mathrm{C}$ for $3 \mathrm{~h}$ and placed on microscope slides. Slides were left overnight at RT and then stained with quinacrine mustard solution (DDK Italia, Milan, Italy) for $3 \mathrm{~h}$. Metaphase pictures were acquired using a Leica DM5000B fluorescent microscope and karyotype reconstructed using Leica Chantal software (Leica Microsystems Srl, Milan, Italy).

\section{hASC characterization}

The hASC characterization was performed by inverted phase contrast microscopy and flow cytometry analysis, as described by Cherubino et al. [2], and immunostaining and quantitative PCR, as reported by Borgese et al. [53]. For cytofluorimetric analysis, the authors used a series of monoclonal antibodies specific for staminal markers CD44, CD90 and CD105; differentiation marker CD45; and major histocompatibility molecules HLA class I (HLA$\mathrm{A}, \mathrm{B}, \mathrm{C})$ and II (HLA-DR). For immunostaining, CD44 antibody was used as a stemness marker and ADIPOR1 antibody as an adipogenic differentiation marker [53].

For quantitative PCR, CD44 and CD90 genes were used as positive stemness markers, whereas FABP4, ACRP30 and ACSS2 genes were taken as differentiation markers. According to the method described by Palombella et al. [52], $G A P D H$ and $\beta 2 M$ were used as reference genes. The relative quantification was calculated using the $2^{-\Delta \Delta \mathrm{Ct}}$ method as previously reported $[2,52]$.

\section{hASC-conditioned medium}

The hASCs were cultured as previously described until passage five. When the hASCs reached $70-80 \%$ confluence, the medium was removed, and the cells were washed twice with PBS and maintained for $48 \mathrm{~h}$ in FBS-free DMEM (serum starvation). The CM, derived from three independent hASC donor cultures, was then collected, centrifuged at $3000 \mathrm{RCF}$ for $10 \mathrm{~min}$ to avoid contamination of cell fragments and stored at $-80^{\circ} \mathrm{C}$ until use [54]. The paracrine effects of the three different hASC-CMs were separately evaluated and, to reduce the variability inherent in primary hASC cultures derived from different subjects, results were expressed as mean \pm standard error (SE).

\section{ELISA on hASC-CM}

To evaluate the proteins secreted by hASCs, an ELISA (BioVendor, Brno, Czech Republic) was performed on the CM following the manufacturer's instructions. The most common pro-angiogenic VEGFA [55] and prolymphangiogenic VEGFC [37] factors, as well as the pleiotropic cytokine IL6 [56], were evaluated. Data were pooled and expressed as mean $\pm \mathrm{SE}$. 
Table 1. Experimental conditions used for LEC growth.

\begin{tabular}{|ll|}
\hline DMEM $_{0.1} \%$ & Culture media \\
\hline DMEM $_{10 \%}$ & DMEM + 0.1\% FBS \\
\hline hASC-CM & DMEM + $10 \%$ FBS \\
\hline $\begin{array}{l}\text { DMEM: Dulbecco's modified Eagle medium; FBS: Fetal bovine serum; hASC-CM: Human adipose-derived stem cell-conditioned medium; LEC: Lymphatic } \\
\text { endothelial cell. }\end{array}$ \\
\hline
\end{tabular}

Rat diaphragmatic LEC isolation, culture \& characterization

All the experiments involving animals were approved by the University of Insubria Ethical Committee and by the Italian Ministry of Health in accordance with the Italian D.Lgs 26/2014. Experiments were performed on adult Wistar rats of both sexes ( $\mathrm{n}=12$; age: $2-12$ months; body weight: $321 \pm 44 \mathrm{~g})$ deeply anesthetized with an intraperitoneal injection of $75 \mathrm{mg} / \mathrm{kg}$ ketamine (Lobotor; Acme Italia Srl, Milan, Italy) and $0.5 \mathrm{mg} / \mathrm{kg}$ medetomidine (Domitor; Pfizer Italia s.r.l., Milan, Italy) cocktail in saline. In vivo fluorescent staining of the pleural diaphragmatic lymphatic network was performed as previously described [25]. Briefly, diaphragmatic lymphatics were stained in vivo by an intraperitoneal injection of $0.8 \mathrm{ml} 2 \%$ fluorescein isothiocyanate-conjugated highmolecular-weight $(250 \mathrm{kDa})$ dextran (FD250S; Sigma-Aldrich) in saline solution, allowing a precise discrimination between lymphatic and blood vessels, as the latter appeared darker and not fluorescent. After $60 \mathrm{~min}$, animals were tracheotomized, paralyzed with a single bolus of $2 \mathrm{mg} / \mathrm{ml}$ pancuronium bromide (P1918; Merck, Italy) in saline solution administered through the jugular vein and mechanically ventilated (Harvard Apparatus Inspira, Vanden Abeele, QC, Canada). The chest wall was then opened, the pleural diaphragmatic surface was exposed and the fluorescein isothiocyanate-filled lymphatic network was visualized under a stereo microscope (SV11; Carl Zeiss S.p.A., Milan, Italy) equipped with light-emitting diode epifluorescence. Then, immediately after animal suppression, fluorescent diaphragmatic lymphatics (microlymphatic vessel mean diameter approximately $150 \mu \mathrm{m}$ ) belonging to superficial collecting lymphatics, mainly located at the far muscle periphery of the costal margin, were carefully dissected from surrounding tissue. Smaller lymphatic capillaries and vessels belonging to lymphatic lacunae appeared fainter and were not considered. Excised lymphatics were placed on 2D, thin, collagen-coated Petri dishes (354236; BD Biosciences, CA, USA) of $50 \mu \mathrm{g} / \mathrm{ml}$ and cultured in DMEM supplemented with 10\% FBS and 1\% penicillin-streptomycin. To avoid contamination of LEC cultures by lymphatic smooth muscle cells or other connective tissue cells, $50 \mu \mathrm{M}$ dibutyryl-cAMP [57] and $1 \mu \mathrm{g} / \mathrm{ml}$ hydrocortisone acetate [58] were added to the growing medium and then maintained for the first growing step. LECs were maintained at $37^{\circ} \mathrm{C}$ and $5 \%$ $\mathrm{CO}_{2}$, and culture medium was changed every 3 days of culture.

Primary LECs started to spread out from tissue samples after about 3 days of culture, showing the typical cobblestone LEC morphology [59-61]. Once LECs reached an adequate number, lymphatic tissue specimens were carefully removed from the culture dish, and the cells were left to grow up to a 70-80\% subconfluent monolayer. LEC lymphatic profile was assayed by immunostaining using the typical lymphatic markers: LYVE1, the membrane glycoprotein podoplanin and the transcription factor PROX1, as reported by Marcozzi et al. [61]. LEC cultures were also verified to be free of lymphatic smooth muscle cell contamination by lymphatic smooth muscle actin immunostaining (data not shown).

\section{LEC treatment with hASC-CM}

Primary diaphragmatic LEC cultures obtained from 12 rats were cultured separately ( $\mathrm{n}=12$ primary LEC cultures) and grown until they reached $70-80 \%$ confluence (passage zero). LEC cultures were then maintained for $24 \mathrm{~h}$ in $\mathrm{DMEM}_{0.1 \%}$ (serum starvation). After serum starvation, images of Petri dishes were captured and then LECs were manually counted to assess the starting conditions ( $\mathrm{t} 0$ ). For each LEC culture, the Petri dishes were randomly divided into different groups and maintained for $48 \mathrm{~h}$ (t48) in culture conditions as controls or hASC-CM treatment, as reported in Table 1. For each individual hASC-CM treatment, four primary LEC cultures were randomly exposed to hASC-CM \#1, hASC-CM \#2 or hASC-CM \#3 individually. For any LEC culture, three Petri dishes were tested for the same hASC-CM, and four fields per hASC-CM were manually counted (total fields $=48$ for each hASC-CM) using the ImageJ software (NIH, MD, USA) Cell Counter plugin (https://imagej.nih.gov/ij/plugins/cell-counter.html) [62]. The resulting cell number was normalized per area unit $\left(\right.$ cells $/ \mathrm{mm}^{2}$ ) to obtain cell density. The cell counting at t0 and t 48 was not intended to evaluate cell proliferation but 
Table 2. Primers used in this work.

\begin{tabular}{|c|c|c|c|}
\hline Gene name & Accession number & Primer sequence $5^{\prime}-3^{\prime}$ & $\begin{array}{l}\text { Melting } \\
\text { temperature } \\
\left({ }^{\circ} \mathrm{C}\right)\end{array}$ \\
\hline \multirow{2}{*}{$\mathrm{Rn} \_G A P D H$} & AF106860.2 & Fw CATCACCATCTTCCAGGA & 59.8 \\
\hline & & Rev GACTCCACGACATACTCA & 59.5 \\
\hline \multirow[t]{2}{*}{$\mathrm{Rn} \_\beta 2 M$} & NM_012512.2 & Fw GGTGACCGTGATCTTTCT & 60.4 \\
\hline & & Rev GGCGAGAGTACACTTGAA & 60.4 \\
\hline \multirow[t]{2}{*}{$\mathrm{Rn} \_R P L 13$} & NM_031101.1 & Fw GAGGTGCCCTACAGTTAG & 60.2 \\
\hline & & Rev TTCTTGTGGATACCAGCC & 60.5 \\
\hline \multirow[t]{2}{*}{$\mathrm{Rn} \_P R O X 1$} & NM_00107201.1 & Fw CCAAGGTTCAGAGCAGGATG & 60.8 \\
\hline & & Rev CATGGCATCTTCATACGAGTTC & 59.6 \\
\hline \multirow[t]{2}{*}{ Rn_VEGFR3 } & AF402786.1 & Fw GTCTTCTTCTGGGTCCTCCTCC & 62.7 \\
\hline & & Rev GGGTCCATGATGATGGACAG & 61.2 \\
\hline \multirow[t]{2}{*}{ Hs_VEGFA ${ }^{\dagger}$} & NM_001025366.2 & Fw CAAGTGGTCCCAGGCTGC & 62.9 \\
\hline & & Rev CTGGAAGATGTCCACCAGGG & 62.8 \\
\hline \multirow[t]{2}{*}{ Hs_VEGFC ${ }^{\dagger}$} & NM_005429.4 & FW TGTGTCCGTCTACAGATGT & 61.9 \\
\hline & & Rev GGCAGGAAGTGTGATTGG & 62.2 \\
\hline \multirow[t]{2}{*}{$\mathrm{Hs}\lrcorner / L \sigma^{\dagger}$} & M14584.1 & Fw ACTCACCTCTTCAGAACG & 60.4 \\
\hline & & Rev CCTCTTTGCTGCTTTCAC & 60.0 \\
\hline
\end{tabular}

to assess baseline reference conditions to point out the hASC-CM paracrine effect following treatment. Moreover, primary LEC cultures developed from the same rats were used as controls and randomly grown in either $\mathrm{DMEM}_{0.1 \%}$ $(n=5)$ or $D_{M E M} \%(n=5)$ for 48 h. For each control, two Petri dishes from five LEC cultures were used, and two fields per culture were manually counted ( $n=20$ total fields).

\section{RNA extraction \& quantitative PCR analysis}

Total LEC mRNA was isolated using the Miniprep kit (Zymo Research, Italy) according to the manufacturer's protocol. The extracted RNA was quantified using the QuantiFluor ${ }^{\circledR}$ RNA system (Promega, WI, USA), and its quality and integrity were assessed by $1 \%$ agarose gel electrophoresis. The RNA was reverse transcribed using the $\mathrm{iScript}^{\mathrm{TM}} \mathrm{cDNA}$ synthesis kit (Bio-Rad, Segrate, Italy), and the $\mathrm{cDNA}$ was stored at $-20^{\circ} \mathrm{C}$ until use.

Quantitative PCR was performed using iTaq ${ }^{T M}$ universal SYBR ${ }^{\circledR}$ green supermix (Bio-Rad). The Beacon Designer program (Bio-Rad) was used to design specific primers (Table 2).

Each sample was prepared as reported by Rossi et al. [63]. Briefly, $1 \mu \mathrm{l}$ (5 ng) of cDNA, $1 \mu$ l of forward and reverse primer mix $(6 \mu \mathrm{M}), 7.5 \mu \mathrm{l}$ of $\operatorname{SYBR} \cap$ green supermix $(2 \mathrm{x})$ and water to a final volume of $15 \mu \mathrm{l}$ were run in the CFX96 thermal cycler (Bio-Rad). Thermal cycle provided the following settings: $5 \mathrm{~min}$ at $95^{\circ} \mathrm{C}, 10$ seconds at $95^{\circ} \mathrm{C}$ and 30 seconds at $60^{\circ} \mathrm{C}$ for 40 cycles. Each experiment was performed in triplicate, and results were normalized with three reference genes, GAPDH, $\beta 2 M$ and RPL13, according to the method described by Palombella et al. [52] and quantified using the $2^{-\Delta \Delta \mathrm{Ct}}$ method.

The lymphatic master regulatory gene, $P R O X 1$ [64], and VEGFR3 were selected as lymphatic markers, whereas $V E G F C$, VEGFA and IL6 were chosen as pro-lymphangiogenic and/or pro-angiogenic indicators. Data for hASCCM-treated LECs were normalized with respect to gene expression in cells grown in $\mathrm{DMEM}_{10 \%}$ medium.

\section{Analysis of LEC morphology \& cell density}

To evaluate morphology and cell density for LECs maintained in $\mathrm{DMEM}_{0.1 \%}$ and $\mathrm{DMEM}_{10 \%}, 20$ fields from five randomly selected Petri dishes were considered. For hASC-CM-treated LECs and for the samples at t0, 48 fields from 12 different replicates were examined. The fields were manually counted as described earlier, and the resulting cell number was normalized per area unit $\left(\right.$ cells $\left./ \mathrm{mm}^{2}\right)$. Freehand selections, corresponding to $2 \mathrm{D}$ culture vessel-like structure (VLS) areas, were measured using ImageJ software (NIH). 
(A)

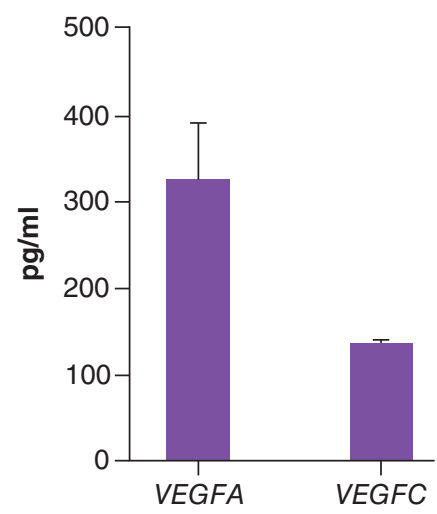

(B)

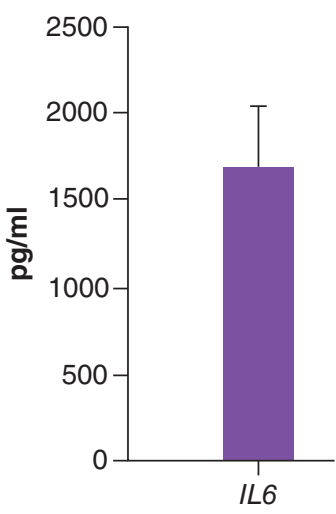

Figure 1. ELISA quantification of VEGFA, VEGFC and IL6. (A) VEGFA, VEGFC protein evaluation in human adipose-derived stem cell-conditioned medium. (B) IL6 protein evaluation in human adipose-derived stem cell-conditioned medium. Results given in $\mathrm{pg} / \mathrm{ml}$, are expressed as mean \pm SE. $\mathrm{n}=3$ subjects.

\section{LEC immunostaining for LYVE1 antibody}

To assess the lymphatic nature of newly formed VLS areas, LECs cultured in hASC-CM for $48 \mathrm{~h}$ were immunostained with the specific lymphatic marker LYVE1 [65], a membrane protein that better identifies the bays of VLS areas. Briefly, LECs were fixed with 4\% paraformaldehyde, washed in PBS and permeabilized with ice-cold methanol and subsequently with $0.5 \%$ Triton X-100 (Sigma-Aldrich) in PBS. After $1 \mathrm{~h}$ in blocking solution (1\% bovine serum albumin plus 5\% goat serum in PBS) at RT, LECs were incubated overnight with anti-LYVE1 antibody LYVE-1 (P-14) (sc-31290; Santa Cruz Biotechnology, Inc., Heidelberg, Germany) at $4{ }^{\circ} \mathrm{C}$. Samples were washed in PBS and then incubated $2 \mathrm{~h}$ with anti-goat secondary antibody conjugated with Texas Red (sc-2783; Santa Cruz Biotechnology, Inc.) at RT, in the dark. Finally, LECs were washed in PBS and mounted with Fluoroshield with 4',6-diamidino-2-phenylindole (F6057; Sigma-Aldrich), and images were collected using an IX51 microscope (Olympus Italia, Milan, Italy).

\section{Statistical analysis}

Statistical analysis was performed using SigmaPlot 10 (Systat Software, Inc., CA, USA) using paired or unpaired Student's t-test and analysis of variance after data normality distribution check. Data were expressed as mean values \pm SE and were considered significantly different at $\mathrm{p}<0.05$.

\section{Results}

hASC characterization

The hASCs used in this study, previously characterized by flow cytometry and immunostaining [2,53], displayed the classic spindle-shaped aspect with fibroblast-like morphology (Supplementary Figure 1). Furthermore, they presented a high capacity for adhesion to the culture flasks and high proliferation potential and exhibited the same immunophenotypic profile of those previously used (Supplementary Figure 2) [53]. In addition, up to the 12th passage, hASCs showed a normal karyotype, confirming the genomic stability essential for gene expression and safety (Supplementary Figure 3) [66].

\section{ELISA on hASC-CM}

ELISA was performed on each CM from hASCs obtained from three different subjects, then data were pooled and expressed as mean $\pm S E$ (Figure 1). Results showed that the amount of VEGFC $(135.4 \pm 4.8 \mathrm{pg} / \mathrm{ml})$ was comparable in the analyzed samples, whereas the amount of VEGFA $(328.6 \pm 71.4 \mathrm{pg} / \mathrm{ml})$ and IL6 $(1642.8 \pm 357.1 \mathrm{pg} / \mathrm{ml})$ was variable among individuals.

\section{Effect of hASC-CM on LEC culture}

LEC morphology at t0 and t48 is shown in Figure 2. In detail, LEC morphology evaluated at t0, after serum starvation for $24 \mathrm{~h}$, as baseline condition, is displayed in panels A, C and E. Morphology of LECs after $48 \mathrm{~h}$ of treatment $(\mathrm{t} 48)$ in $\mathrm{DMEM}_{0.1 \%}$ or DMEM $\mathrm{DM}_{10 \%}$ is represented in panels $\mathrm{B}$ and D. By contrast, LECs in hASC-CM are shown in panels $\mathrm{F}$ and $\mathrm{G}$. It is worth noting that at t48, LECs cultured in $\mathrm{DMEM}_{0.1 \%}$ and $\mathrm{DMEM}_{10 \%}$ seemed to lose the typical endothelial cobblestone morphology, whereas hELISA quantification of VEGFA,VEGFC (panel a) and IL6ASC-CM-treated LECs maintained their correct morphology. In addition, at t48, only hASC-CM-treated 
to

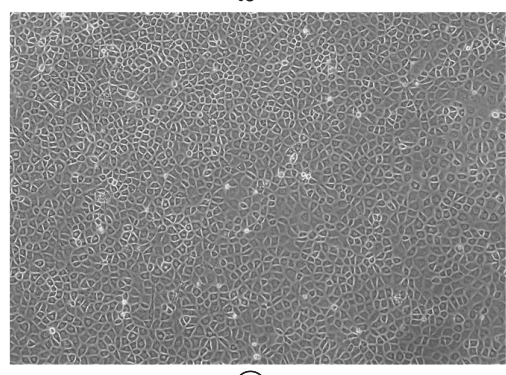

(A)

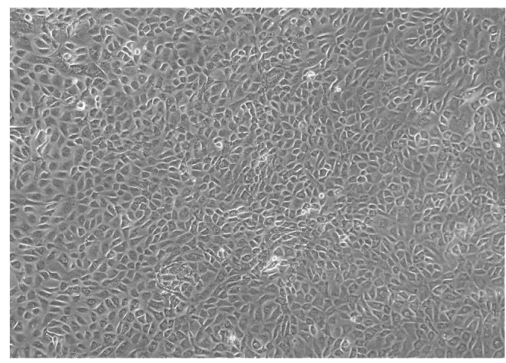

(C)

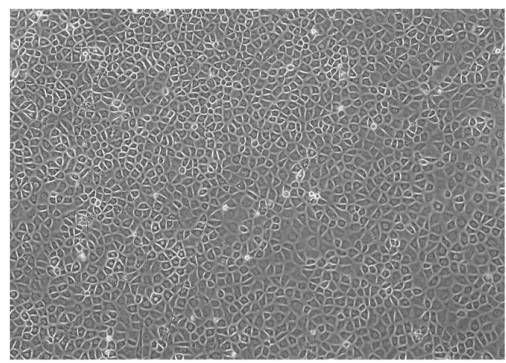

(E) $\mathrm{t} 48 \mathrm{~h}$

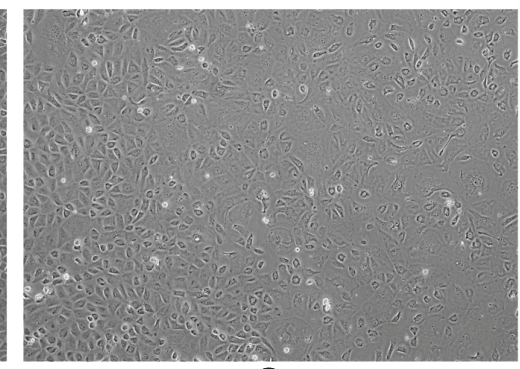

(B)

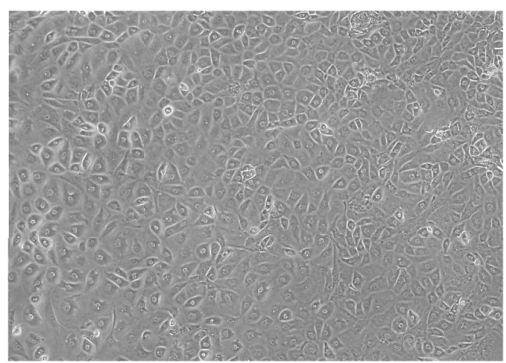

(D)

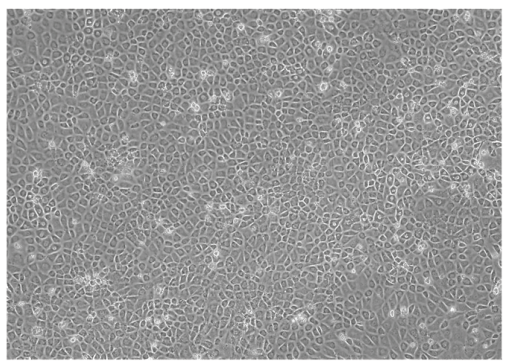

(F)

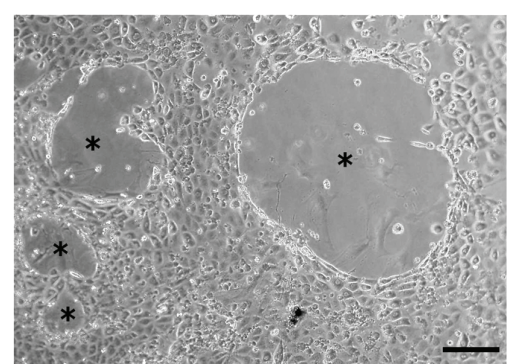

(G)

Figure 2. Effect of human adipose-derived stem cell-conditioned medium treatment on lymphatic endothelial cell culture. (A, C \& E) Representative images of cells after $24 \mathrm{~h}$ of serum starvation (t0). (B) Cells maintained in Dulbecco's modified Eagle medium (DMEM) $)_{0.1 \%}$ for $48 \mathrm{~h}$ of culture (t48). (D) Cells maintained for $48 \mathrm{~h}$ of culture in $\mathrm{DMEM}_{10 \%}$. (F \& G) Cells maintained for $48 \mathrm{~h}$ of culture in human adipose-derived stem cell-conditioned medium. (G) Lymphatic endothelial cells maintained in human adipose-derived stem cell-conditioned medium were able to organize vessel-like structure (*). Scalebar $200 \mu \mathrm{m}$.

LECs showed the predisposition to form VLS areas in $2 \mathrm{D}$ cell cultures. The mean area covered by the branching points of these structures was measured in different fields and was about $1.53^{*} 10^{5} \pm 4.5^{*} 10^{4} \mu \mathrm{m}^{2}$ ( $\mathrm{n}=16 \mathrm{VLS}$ areas).

Results on cell density are depicted in Figure 3. The cell counting assay was intended to measure hASC-CM effect on LEC survival and possible role in prevention of cell dedifferentiation. After serum starvation for $24 \mathrm{~h}(\mathrm{t} 0)$, LEC density was found to be similar in all samples $(\mathrm{p}=0.87)$ and was $1129 \pm 71 \mathrm{cells} / \mathrm{mm}^{2}$ for $\mathrm{DMEM}_{0.1 \%}$, $1116 \pm 57$ cells $/ \mathrm{mm}^{2}$ for $\mathrm{DMEM}_{10 \%}$ and $1167 \pm 66$ cells $/ \mathrm{mm}^{2}$ for LECs subsequently treated with hASC-CM. At t48, LECs grown in $\mathrm{DMEM}_{0.1 \%}$ showed a very significant decrease $(\sim 90 \%)$ in cell density $\left(138 \pm 6 \mathrm{cells} / \mathrm{mm}^{2}\right.$ vs corresponding t0; $\mathrm{p}<0.01 ; \mathrm{n}=5)$. Moreover, at $\mathrm{t} 48$, even LECs grown in the standard condition $\mathrm{DMEM}_{10 \%}$ showed a significant decrease $(\sim 50 \%)$ in cell density $\left(553 \pm 49\right.$ cells $/ \mathrm{mm}^{2}$ vs corresponding t $\left.0 ; \mathrm{p}<0.01 ; \mathrm{n}=5\right)$. By contrast, LECs maintained in hASC-CM for $48 \mathrm{~h}$ showed a slight, although not significant, increase in cell density $\left(1303 \pm 114\right.$ cells $/ \mathrm{mm}^{2}$ vs corresponding t0). Furthermore, hASC-CM had a major beneficial effect on LECs compared with those maintained in the standard condition DMEM DM\% $_{10}\left(1303 \pm 114\right.$ cells $/ \mathrm{mm}^{2}$ for hASC-CM vs $553 \pm 49$ cells $/ \mathrm{mm}^{2}$ for $\left.\mathrm{DMEM}_{10 \%} ; \mathrm{p}<0.01 ; \mathrm{n}=17\right)$. This effect was even more evident when compared with LECs grown in $\mathrm{DMEM}_{0.1 \%}(\mathrm{p}<0.01 ; \mathrm{n}=17)$.

Figure 4 shows the effect for hASC-CM \# 1, hASC-CM \#2 and hASC-CM \#3 at t 48 with respect to t0. All hASCconditioned media exerted a positive paracrine effect on cultured LECs, promoting cell survival, maintaining LEC 


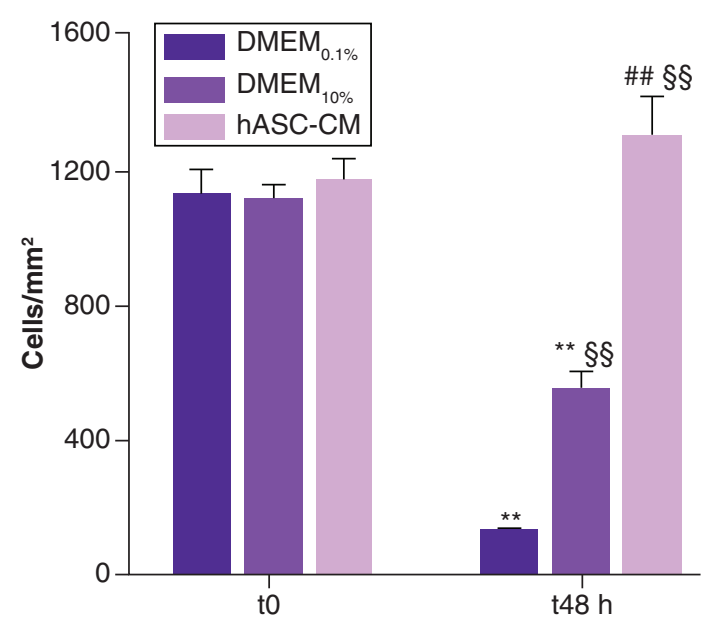

Figure 3. Evaluation of lymphatic endothelial cell density. Cells, expressed as number of cells $/ \mathrm{mm}^{2}$, are counted at t0, baseline condition, and t48. For $\mathrm{DMEM}_{0.1 \%}$ and $\mathrm{DMEM}_{10 \%}$, 20 fields/each were evaluated; for hASC-CM, 48 fields/each were analyzed.

**Data statistically significant, $p<0.01$, paired t-test t48 vs proper t0 $\mathrm{n}=5$ for $\mathrm{DMEM}_{0.1 \%}$ and $\mathrm{DMEM}_{10 \%}, \mathrm{n}=12$ for hASC-CM.

$\S \S$ Data statistically significant, $\mathrm{p}<0.01$, unpaired t-test vs DMEM $_{0.1 \%}$ t48 h, $\mathrm{n}=17$.

\#\#Data statistically significant, $p<0.01$, unpaired t-test vs DMEM $_{10 \%}$ t48 $\mathrm{h} \mathrm{n}=17$.

DMEM: Dulbecco's modified Eagle medium; hASC-CM: Human adipose-derived stem cell conditioned medium.

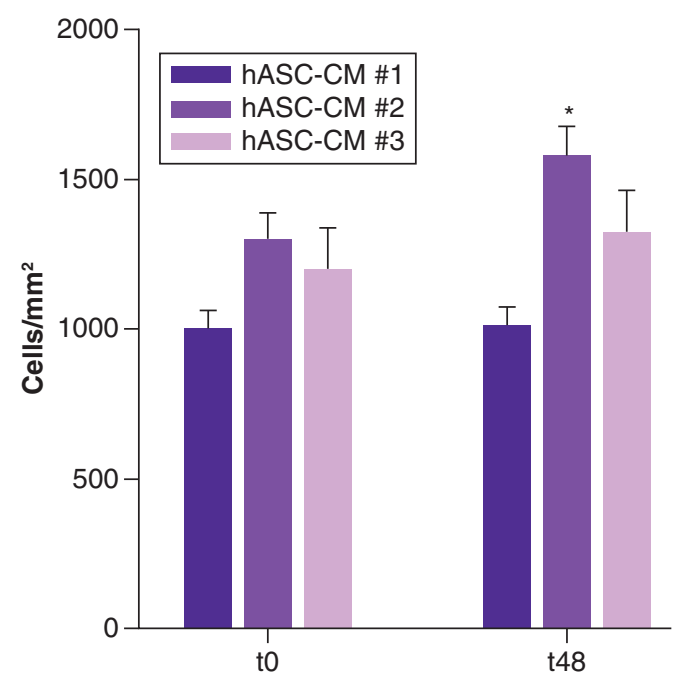

Figure 4. Evaluation of lymphatic endothelial cell density. hASC-CM: Human adipose-derived stem cell conditioned medium.

$* p<0.05$.

phenotype and preventing cell dedifferentiation. Only hASC-CM \#2 was able to induce an increase ( $\mathrm{p}<0.05$; $\mathrm{n}=4$ ) in cell density at $\mathrm{t} 48$.

To confirm the lymphatic nature of the 2D newly formed VLS area, an immunostaining assay was performed using the specific lymphatic marker anti-LYVE1 antibody. As reported in Figure 5, red positively stained cells confirmed the lymphatic features of those new structures developed from organized LECs. As shown in inset a', corresponding to the square of the main panel, the red signal delimited the borders of VLS. Inset a' (corresponding to higher magnification of square a) highlights the red staining delimiting the borders of VLS.

\section{Quantitative PCR on LECs treated with hASC-CM}

Figure 6 represents the relative gene expression of lymphatic markers (PROX1 and VEGFR3), growth factors (VEGFC and VEGFA) and pleiotropic cytokine IL6 evaluated at t48 in LECs maintained in $\mathrm{DMEM}_{10 \%}$ or grown in hASC-CM. Considering that hASC-CM, grown in $\mathrm{DMEM}_{0.1 \%} \mathrm{FBS}$, resulted in an extreme cell density reduction $(\sim-90 \%)$ in $48 \mathrm{~h}$, this condition was not suitable for performing gene expression comparison; consequently, the authors decided to consider $\mathrm{DMEM}_{10 \%}$, the optimal standard growing condition, the control. Data showed that hASC-CM treatment induced a significant increase in the expression of the lymphatic markers PROXI ( $\sim$ threefold vs $\mathrm{DMEM}_{10 \%} ; \mathrm{p}<0.01 ; \mathrm{n}=12$ ) and VEGFR3 ( fivefold vs $\mathrm{DMEM}_{10 \%} ; \mathrm{p}<0.05 ; \mathrm{n}=12$ ). An interesting, although not significant, decrease in the expression of $V E G F C\left(\sim-90 \%\right.$ vs $\left.\mathrm{DMEM}_{10 \%}\right)$ was also found. Conversely, no significant differences were found for the expression of VEGFA and ILG. 


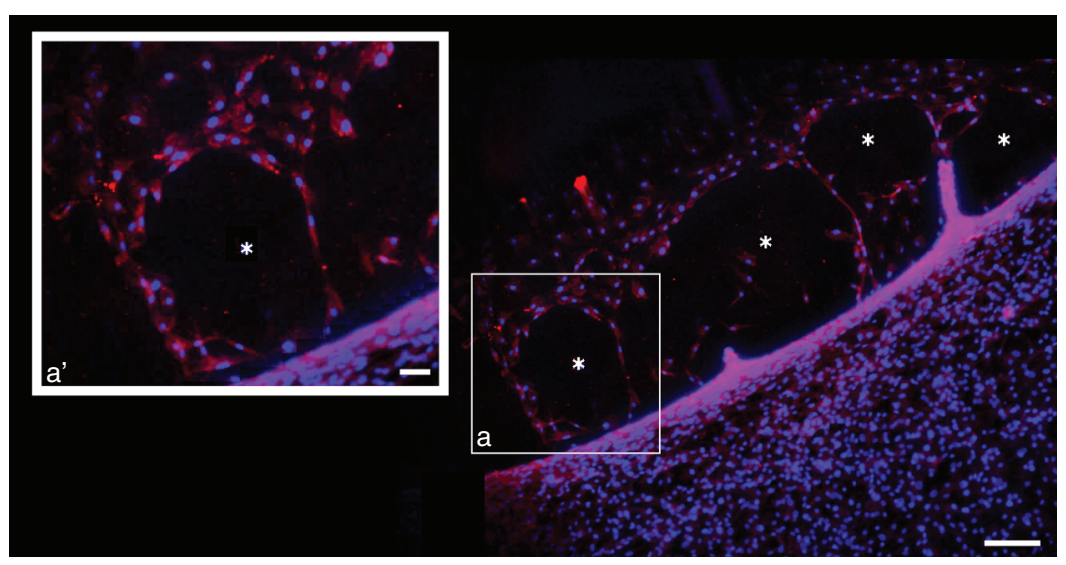

Figure 5. Immunostaining assay of lymphatic endothelial cells and vessel like structures. Representative image of positive LYVE1 expressing cells (red staining) in t48 human adipose-derived stem cell conditioned medium treated lymphatic endothelial cells. Blue DAPI staining indicates nuclei. (a') shows higher magnification of (a), which highlights the red staining delimiting the borders of vessel-like structure.

* = newly formed vessel-like structure.

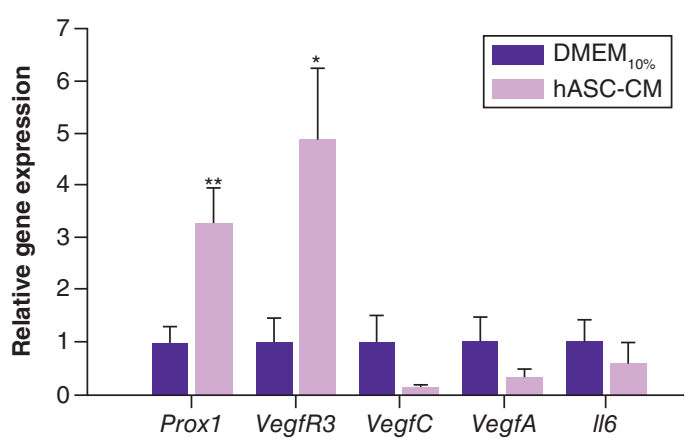

Figure 6. Gene expression evaluation. Prox1 and VegfR3 expression were significantly upregulated by hASC-CM treatment.

${ }^{* *} \mathrm{p}<0.01$ and ${ }^{*} \mathrm{p}<0.05$, respectively vs $\mathrm{DMEM}_{10 \%}$, unpaired t-test, $\mathrm{n}=12$.

DMEM: Dulbecco's modified Eagle medium; hASC-CM: Human adipose-derived stem cell conditioned medium.

\section{Discussion}

Aberrant lymphatic vessel growth and lymphatic vessel dysfunction are associated with pathological conditions such as inflammation, lymphedema and tumor metastasis; therefore, lymphatic vessels are an attractive target for therapeutic intervention. Secondary lymphedema occurs as a result of damage to the lymphatic system, typically after breast or pelvic surgery. No cure has been established, and current therapeutic options remain limited and largely ineffective.

An intricate balance of growth factors and biomechanical cues controls the mechanisms by which the lymphatic vasculature network is formed, remodeled and adapted to physiological and pathological conditions [67,68]. In this context, the role of hASCs has been extensively demonstrated in regenerative medicine, but thus far their paracrine effect on lymphatic vessels remains incompletely understood. The evidence supports the hypothesis that growth factors, secreted by hASCs, could represent an easily accessible source of pro-lymphangiogenic molecules, able to bring clinical benefits to the treatment of secondary lymphedema $[40-42,49]$.

In this work, the authors evaluated the effects of the bioactive molecules released by hASCs, using their culture medium to grow primary rat diaphragmatic LECs. These LECs, even deriving from a different source tissue and organ, showed typical cobblestone morphology and express characteristic markers also found in other primary and commercial cell lines. ELISA of hASC-CM highlighted that hASCs released VEGFC (Figure 1A), a growth factor heavily involved in the mechanisms underlying proper lymphatic function.

Recent evidence has demonstrated that exogenous VEGFC administration significantly improves edema resolution by increasing the growth of functional lymphatic capillaries [69,70] and collecting vessels [35], supporting the key role of the VEGFC/VEGFR3 signaling pathway in lymphangiogenesis [38,46,69]. As reported by Takeda et al. [41], culturing human dermal LECs with murine ASC-CM results in better cell proliferation, migration and tube formation than basal medium supplemented with VEGFC alone [70]. Moreover, hASC-CM proves to be 
even more potent in in vitro lymphangiogenesis assays, promoting proliferation, migration, tube formation and sprouting [42].

The cell density analysis aimed to evaluate whether hASC-CM had any positive effect on LEC maintenance and could prevent cell dedifferentiation. The authors' experimental conditions confirmed the beneficial effect of hASC-secreted factors on LEC survival, morphology maintenance and integrity, even compared with the standard growing condition $\mathrm{DMEM}_{10 \%}$ (Figure 2D \& F). Moreover, LECs cultured for $48 \mathrm{~h}$ in $\mathrm{DMEM}_{0.1 \%}$ showed a significant reduction in cell density $(\sim 90 \%)$ (Figure 3 ), probably due to a high level of cell mortality and/or cell differentiation reinforcing the significant positive effect exerted by hASC-CM on cultured LECs. The positive effect exerted on LEC density was also evident when the three hASC-conditioned media were individually tested (Figure 4). However, considering all data together as a unique hASC-CM treatment (Figure 3), the overall slight increase was not significant. Results suggest a promising role for hASC-CM as a whole, as a combination of growing factors, in developing new therapeutic approaches for lymphatic system-related dysfunction. Similar to what was reported by Podgrabinska et al. [71], treatment with hASC-CM promoted LEC organization in VLS areas (Figure 2G) in 2D cultures, suggesting that VEGFC in the hASC-CM is crucial for LEC organization. LECs exposed to hASC-CM within $48 \mathrm{~h}$ were able to line up, forming round structures (VLS areas) delimited by elongated LECs, though these structures were far from functional. Although hASC-released growing factors provided an adequate environment for inducing LECs to form vessel-like structures in vitro, further experiments will be required to confirm the ability of LECs to organize 3D tube-like structures, via the use of a collagen gel "sandwich" assay or Matrigel ${ }^{\circledR}$ (Thermo Fisher Scientific Inc. Monza MB, Italy), when exposed to hASC-CM. Immunofluorescence results confirmed the lymphatic nature of newly formed VLS areas derived from hASC-CM-treated LEC cultures (Figure 5).

Quantitative PCR analysis indicated that the gene expression of PROX1, the lymphatic master regulatory gene that drives lymphatic identity and maintenance [72], is promoted in LECs grown in hASC-CM. The same result was also found for VEGFR3, the receptor that responds to VEGFC (Figure 6). These outcomes suggest a possible paracrine effect of hASCs via the release of VEGFC, and this hypothesis is supported by the high reduction in $V E G F C$ gene expression (Figure 6) in LECs after hASC-CM. As reported in the literature, the best known signaling system for lymphangiogenesis involves the secreted glycoprotein VEGFC, which acts via VEGFR3 expressed on the surface of LECs [39,40]. Moreover, VEGFC influences the functional relationship between PROX1 and VEGFR3, establishing a feedback loop necessary to maintain the identity of LECs [73,74]. From this perspective, the authors' results suggest that LECs possess an autocrine VEGFC/VEGFR3 loop that probably becomes redundant and then downregulates with the addition of exogenous VEGFC. Thus, the authors hypothesize that, in these experimental conditions, VEGFC plays a pivotal role in enhancing this regulatory mechanism. Although it is known that IL6 also induces VEGFC expression in LECs [56], the signaling mechanisms involved in IL6-mediated VEGFC induction in LECs remain incompletely understood.

The current evidence indicates that hASC-CM may be used to influence the properties of LECs and improve lymphatic vessel function, and this could be considered one step toward using hASC-CM to improve or prevent lymphatic-related pathologies such as secondary lymphedema. Preliminary results obtained in this work (Figures 2 \& 3) demonstrate the critical role played by hASC-CM as a whole, as a combination of secreted factors, in LECs derived from a particular area of the body, such as the diaphragm-muscular tissue located inside the thermal core. Thus, the use of a combination of complementary growth factors, such as those present in hASC-CM, could be more advantageous compared with the addition of single growth factors or even stem cell transplantation. In this regard, the absence of an hASC-CM immune response could solve some issues and difficulties associated with the use of cell transplants Therefore, hASC-CM could represent an option for therapeutic lymphangiogenesis in pathological conditions. In addition, hASC-CM is easier to maintain and transport, and the secretome can be pooled and stored for long-term use [5,75]. Nevertheless, an in-depth characterization of CM could provide clues as to specific molecules and mechanisms pivotally involved in the paracrine effect exerted on LECs.

\section{Conclusion}

The study provides evidence of an hASC paracrine effect on LECs and emphasizes the advantages of secretome derivatives for stimulating the in vitro lymphangiogenesis process. The authors' results suggest that the major hASC paracrine effect on LECs is probably exerted by enhancing the VEGFC/VEGFR3 lymphatic endothelial loop. Certainly, further studies will be necessary to elucidate the mechanisms by which the hASC secretome influences LEC gene expression, proliferation, migration and organization into capillary-like structures. Nevertheless, the use 
of hASC-CM, as a combination of complementary growth factors, could be an innovative therapeutic approach for regenerative medicine and may have an important impact on the treatment of lymphatic-related pathologies.

\section{Future perspective}

The use of stem cells for both autologous and heterologous transplants might cause ectopic growth, tumorigenesis and immune system reactions. By contrast, the adoption of a cell-free therapy would significantly reduce the risks and overcome the difficulties associated with direct cell injection. The (stem) cell-free therapy is based on biologically active factors secreted by stem cells, and the use of a combination of interdependent growth factors, such as those present in hASC-CM, may have substantial advantages compared with the use of single growth factors.

The stem cell secretome, in addition to avoiding cell therapy side effects, has substantial advantages in manufacture, storage and standardization, making it a promising type of biopharmaceutical. Even though an exhaustive characterization of the components of the conditioned culture medium, which also includes the microvesicle fraction, must be done, this emerging therapeutic treatment is a very encouraging, innovative therapeutic approach for regenerative medicine and may have an important impact on the treatment of lymphatic-related pathologies.

\section{Summary points}

- Human adipose-derived stem cells were used as a model to evaluate the paracrine effect on lymphatic endothelial cells (LECs).

- LECs used in this research were primary cultures isolated from rat diaphragmatic lymphatic vessels.

- The study was conducted using a multidisciplinary approach based on immunohistochemistry, gene expression and ELISA.

- Human adipose-derived stem cell-conditioned medium was able to promote LEC survival and morphology maintenance.

- Human adipose-derived stem cell-conditioned medium has similar effects, in terms of tissue repair processes, to those exerted by the cells when inoculated into animal models.

- The conditioned media, which showed the same behavior, were obtained from cells derived from three different subjects, individually cultured and tested.

- The authors' results suggest that LECs possess an autocrine VEGFC/VEGFR3 loop that probably becomes redundant and then downregulates with the addition of exogenous VEGFC.

- The results presented here are intended to add new information to the body of knowledge regarding the potential of cell-free devices in the therapeutic approach to the treatment of lymphatic-related pathologies.

\section{Supplementary data}

To view the supplementary data that accompany this paper please visit the journal website at: www.futuremedicine.com/doi/sup $\mathrm{pl} / 10.2217 / \mathrm{rme}-2020-0071$

\section{Author contributions}

Conceived and designed the experiments: R Gornati, A Frattini and C Marcozzi. Performed the experiments: C Marcozzi, F Rossi, M Borgese, A Frattini and L Barone. Analyzed the data: R Gornati, C Marcozzi, A Frattini, M Borgese and E Solari. Wrote and edited the manuscript: R Gornati, C Marcozzi, A Frattini and E Solari. Contributed reagents/materials/analysis tools: R Gornati, C Marcozzi and R Valli.

\section{Acknowledgments}

M Borgese is a PhD student in biotechnology, biosciences and surgical technology at the University of Insubria (Italy).

Financial \& competing interests disclosure

This work has been financially supported by Fondo comune di Ateneo per la Ricerca (FAR2016; University of Insubria, Italy) to R Gornati and C Marcozzi. The authors have no other relevant affiliations or financial involvement with any organization or entity with a financial interest in or financial conflict with the subject matter or materials discussed in the manuscript apart from those disclosed.

No writing assistance was utilized in the production of this manuscript. 
Ethical conduct of research

All subjects gave their informed consent to be included in this study. All procedures were performed in accordance with the Ospedale di Circolo Ethical Committee and the European Communities Council Directive of EU/63/2010. The Italian Ministry of Health (192/2013) approved the experimental study.

\section{Open access}

This work is licensed under the Attribution-NonCommercial-NoDerivatives 4.0 Unported License. To view a copy of this license, visit http://creativecommons.org/licenses/by-nc-nd/4.0/

\section{References}

Papers of special note have been highlighted as: $\bullet$ of interest; $\bullet \bullet$ of considerable interest

1. Zuk PA, Zhu M, Ashjian P et al. Human adipose tissue is a source of multipotent stem cells. Mol. Biol. Cell 13(12), 4279-4295 (2002).

2. Cherubino M, Valdatta L, Balzaretti $\mathrm{R}$ et al. Human adipose-derived stem cells promote vascularization of collagen-based scaffolds transplanted into nude mice. Regen. Med. 11(3), 261-271 (2016).

3. Gimble JM, Guilak F. Differentiation potential of adipose derived adult stem (ADAS) cells. Curr. Top. Dev. Biol. 58, 137-160 (2003).

4. Yu G, Floyd ZE, Wu X et al. Adipogenic differentiation of adipose-derived stem cells. Methods Mol. Biol. 702, 193-200 (2011).

5. Dubey NK, Mishra VK, Dubey R, Deng YH, Tsai FC, Deng WP. Revisiting the advances in isolation, characterization and secretome of adipose-derived stromal/stem cells. Int. J. Mol. Sci. 19(8), 2200 (2018).

6. Salgado AJ, Reis RL, Sousa NJ, Gimble JM. Adipose tissue derived stem cells secretome: soluble factors and their roles in regenerative medicine. Curr. Stem Cell Res. Ther. 5(2), 103-110 (2010).

7. Niada S, Giannasi C, Gualerzi A, Banfi G, Brini AT. Differential proteomic analysis predicts appropriate applications for the secretome of adipose-derived mesenchymal stem/stromal cells and dermal fibroblasts. Stem Cells Int. 2018, 7309031 (2018).

-. Helps in understanding the importance of the secretome in medical applications.

8. Mirotsou M, Jayawardena TM, Schmeckpeper J, Gnecchi M, Dzau VJ. Paracrine mechanisms of stem cell reparative and regenerative actions in the heart. J. Mol. Cell. Cardiol. 50(2), 280-289 (2011).

9. Suga H, Glotzbach JP, Sorkin M, Longaker MT, Gurtner GC. Paracrine mechanism of angiogenesis in adipose-derived stem cell transplantation. Ann. Plast. Surg. 72(2), 234-241 (2014).

10. Souza LEB, Beckenkamp LR, Sobral LM et al. Pre-culture in endothelial growth medium enhances the angiogenic properties of adipose-derived stem/stromal cells. Angiogenesis 21(1), 15-22 (2018).

11. Rehman J, Traktuev D, Li J, Merfeld-Clauss S et al. Secretion of angiogenic and antiapoptotic factors by human adipose stromal cells. Circulation 109(10), 1292-1298 (2004).

12. Kilroy GE, Foster SJ, Wu X et al. Cytokine profile of human adipose-derived stem cells: expression of angiogenic, hematopoietic, and pro-inflammatory factors. J. Cell. Physiol. 212(3), 702-709 (2007).

13. Nakanishi C, Nagaya N, Ohnishi $\mathrm{S}$ et al. Gene and protein expression analysis of mesenchymal stem cells derived from rat adipose tissue and bone marrow. Circ. J. 75(9), 2260-2268 (2011).

14. Gnecchi M, Zhang MZ, Ni A Dzau VJ. Paracrine mechanisms in adult stem cell signaling and therapy. Circ. Res. 103, 1204-1219 (2008).

-. Helps in understanding the paracrine mechanisms.

15. Egashira Y, Sugitani S, Suzuki Y et al. The conditioned medium of murine and human adipose-derived stem cells exerts neuroprotective effects against experimental stroke model. Brain Res. 1461, 87-95 (2012).

- Discusses protective effect of conditioned medium of human adipose-derived stem cells.

16. Cho YJ, Song HB, Bhang $S$ et al. Therapeutic effects of human adipose stem cell-conditioned medium on stroke. J. Neurosci. Res. 90(9), 1794-1802 (2012).

- Discusses therapeutic effect of conditioned medium of human adipose-derived stem cells.

17. Di Cesare Mannelli L, Tenci B, Micheli L et al. Adipose-derived stem cells decrease pain in a rat model of oxaliplatin-induced neuropathy: role of VEGF-A modulation. Neuropharmacol. 131, 166-175 (2018).

18. Nakagami H, Maeda K, Morishita R et al. Novel autologous cell therapy in ischemic limb disease through growth factor secretion by cultured adipose tissue-derived stromal cells. Arterioscler. Thromb. Vasc. Biol. 25(12), 2542-2547 (2005).

19. Takahashi M, Suzuki E, Oba $S$ et al. Adipose tissue-derived stem cells inhibit neointimal formation in a paracrine fashion in rat femoral artery. Am. J. Physiol. Heart Circ. Physiol. 298(2), H415-H423 (2010).

20. Strassburg S, Torio-Padron N, Finkenzeller G, Frankenschmidt A, Stark GB. Adipose-derived stem cells support lymphangiogenic parameters in vitro. J. Cell. Biochem. 117(11), 2620-2629 (2016).

- Helps in understanding the importance of adipose-derived stem cells in lymphangiogenic promotion. 
21. Moriondo A, Boschetti F, Bianchin F, Lattanzio S, Marcozzi C, Negrini D. Tissue contribution to the mechanical features of diaphragmatic initial lymphatics. J. Physiol. 588(Pt 20), 3957-3969 (2010).

22. Moriondo A, Solari E, Marcozzi C, Negrini D. Lymph flow pattern in pleural diaphragmatic lymphatics during intrinsic and extrinsic isotonic contraction. Am. J. Physiol. Heart Circ. Physiol. 310(1), H60-H70 (2016).

23. Moriondo A, Solari E, Marcozzi C, Negrini D. Diaphragmatic lymphatic vessel behavior during local skeletal muscle contraction. Am. J. Physiol. Heart Circ. Physiol. 308(3), H193-H205 (2015).

24. Muthuchamy M, Gashev A, Boswell N, Dawson N, Zawieja D. Molecular and functional analyses of the contractile apparatus in lymphatic muscle. FASEB J. 17(8), 920-922 (2003).

25. Moriondo A, Solari E, Marcozzi C, Negrini D. Spontaneous activity in peripheral diaphragmatic lymphatic loops. Am. J. Physiol. Heart Circ. Physiol. 305(7), H987-H995 (2013).

26. Negrini D, Marcozzi C, Solari E et al. Hyperpolarization-activated cyclic nucleotide-gated channels in peripheral diaphragmatic lymphatics. Am. J. Physiol. Heart Circ. Physiol. 311(4), H892-H903 (2016).

27. Grada AA, Phillips T. Lymphedema: diagnostic workup and management. J. Am. Acad. Dermatol. 77(6), 995-1006 (2017).

28. Ferrell RE, Levinson K, Esman JH et al. Hereditary lymphedema: evidence for linkage and genetic heterogeneity. Hum. Mol. Genet. 7(13), 2073-2078 (1998).

29. Connell F, Brice G, Mortimer P. Phenotypic characterization of primary lymphedema. Ann. N. Y. Acad. Sci. 1131, 140-146 (2008).

30. Brice G, Child AH, Evans A et al. Milroy disease and the VEGFR-3 mutation phenotype. J. Med. Genet. 42(2), 98-102 (2005).

31. Grada AA, Phillips TJ. Lymphedema: pathophysiology and clinical manifestations. J. Am. Acad. Dermatol. 77(6), 1009-1020 (2017).

32. Oremus M, Dayes I, Walker K, Raina P. Systematic review: conservative treatments for secondary lymphedema. BMC Cancer 12, 1-15 (2012).

33. Yoshida S, Hamuy R, Hamada Y, Yoshimoto H, Hirano A, Akita S. Adipose-derived stem cell transplantation for therapeutic lymphangiogenesis in a mouse secondary lymphedema model. Regen. Med. 10(5), 549-562 (2015).

34. Toyserkani NM, Jensen CH, Andersen DC, Sheikh SP, Sørensen JA. Treatment of breast cancer-related lymphedema with adipose-derived regenerative cells and fat grafts: a feasibility and safety study. Stem Cells Transl. Med. 6(8), 1666-1672 (2017).

35. Joukov V, Pajusola K, Kaipainen A et al. A novel vascular endothelial growth factor, VEGF-C, is a ligand for the Flt4 (VEGFR-3) and KDR (VEGFR-2) receptor tyrosine kinases. EMBO J. 15(2), 290-298 (1996).

36. Jeltsch M, Kaipainen A, Joukov V et al. Hyperplasia of lymphatic vessels in VEGF-C transgenic mice. Science 276(5317), 1423-1425 (1997).

37. Karkkainen MJ, Haiko P, Sainio $\mathrm{K}$ et al. Vascular endothelial growth factor $\mathrm{C}$ is required for sprouting of the first lymphatic vessels from embryonic veins. Nature Immunol. 5(1), 74-80 (2004).

38. Mäkinen T, Veikkola T, Mustjoki $S$ et al. Isolated lymphatic endothelial cells transduce growth, survival and migratory signals via the VEGF-C/D receptor VEGFR-3. EMBO J. 20(17), 4762-4773 (2001).

39. Wang J, Huang Y, Zhang J et al. Pathway-related molecules of VEGFC/D-VEGFR3/NRP2 axis in tumor lymphangiogenesis and lymphatic metastasis. Clin. Chim. Acta 461, 165-171 (2016).

40. Yan A, Avraham T, Zampell JC, Haviv YS, Weitman E, Mehrara BJ. Adipose-derived stem cells promote lymphangiogenesis in response to VEGF-C stimulation or TGF- $\beta 1$ inhibition. Future Oncol. 7(12), 1457-1473 (2011).

41. Takeda K, Sowa Y, Nishino K, Itoh K, Fushiki S. Adipose-derived stem cells promote proliferation, migration, and tube formation of lymphatic endothelial cells in vitro by secreting lymphangiogenic factors. Ann. Plast. Surg. 74(6), 728-736 (2015).

42. Ahmadzadeh N, Robering JW, Kengelbach-Weigand A et al. Human adipose-derived stem cells support lymphangiogenesis in vitro by secretion of lymphangiogenic factors. Exp. Cell. Res. 388(2), 111816 (2020).

43. Makinen T, Jussila T, Veikkola $\mathrm{T}$ et al. Inhibition of lymphangiogenesis with resulting lymphedema in transgenic mice expressing soluble VEGF receptor-3. Nature Med. 7(2), 199-205 (2001).

44. Karkkainen MJ, Ferrell RE, Lawrence EC et al. Missense mutations interfere with VEGFR-3 signaling in primary lymphedema. Nature Genet. 25(2), 153-159 (2000).

45. Irrthum A, Karkkainen MJ, Devriendt K, Alitalo K, Vikkula M. Congenital hereditary lymphedema caused by a mutation that inactivates VEGFR3 tyrosine kinase. Am. J. Hum. Genet. 67(2), 295-301 (2000).

46. Breslin JW, Gaudreault N, Watson KD, Reynoso R, Yuan SY, Wu MH. Vascular endothelial growth factor-C stimulates the lymphatic pump by a VEGF receptor-3-dependent mechanism. Am. J. Physiol. Heart Circ. Physiol. 293(1), H709-H718 (2007).

47. Harvey NL. The link between lymphatic function and adipose biology. Ann. N. Y. Acad. Sci. 1131, 82-88 (2008).

48. Yang Y, Chen XH, Li FG et al. In vitro induction of human adipose-derived stem cells into lymphatic endothelial-like cells. Cell Reprogram. 17(1), 69-76 (2015).

49. Saijo H, Suzuki K, Yoshimoto H, Imamura Y, Yamashita S, Tanaka K. Paracrine effects of adipose-derived stem cells promote lymphangiogenesis in irradiated lymphatic endothelial cells. Plast. Reconstr. Surg. 143(6), 1189e-1200e (2019). 
50. Solari E, Marcozzi C, Negrini D, Moriondo A. Temperature-dependent modulation of regional lymphatic contraction frequency and flow. Am. J. Physiol. Heart Circ. Physiol. 313(5), H879-H889 (2017).

51. Gronthos S, Zannettino ACW. Methods for the purification and characterization of human adipose-derived stem cells. Methods Mol. Biol. 702, 109-120 (2011).

52. Palombella S, Pirrone C, Cherubino M, Valdatta L, Bernardini G, Gornati R. Identification of reference genes for qPCR analysis during hASC long culture maintenance. PLoS ONE 12(2), e0170918 (2017).

53. Borgese M, Rossi F, Bonfanti P et al. Recovery ability of human adipose stem cells exposed to cobalt nanoparticles: outcome of dissolution. Nanomedicine 15(5), 453-465 (2020).

54. Sugitani S, Tsuruma K, Ohno Y et al. The potential neuroprotective effect of human adipose stem cells conditioned medium against light-induced retinal damage. Exp. Eye Res. 116, 254-264 (2013).

55. Shibuya M. Vascular endothelial growth factor (VEGF) and its receptor (VEGFR) signaling in angiogenesis: a crucial target for anti-and pro-angiogenic therapies. Genes Cancer 2(12), 1097-1105 (2011).

56. Huang YH, Yang HY, Huang SW, Ou G, Hsu YF, Hsu MJ. Interleukin-6 induces vascular endothelial growth factor-C expression via Src-FAK-STAT3 signaling in lymphatic endothelial cells. PLoS ONE 11(7), e0158839 (2016).

57. Leszczynski D, Zhao Y, Luokkamäki M, Foegh ML. Apoptosis of vascular smooth muscle cells. Protein kinase C and oncoprotein Bcl-2 are involved in regulation of apoptosis in non-transformed rat vascular smooth muscle cells. Am. J. Pathol. 145(6), 1265-1270 (1994).

58. Holden M, Adams LB. Inhibitory effects of cortisone acetate and hydrocortisone on growth of fibroblasts. Proc. Soc. Exp. Biol. Med. 95(2), 364-368 (1957).

59. Hayes H, Kossmann E, Wilson E, Meininger C, Zawieja D. Development and characterization of endothelial cells from rat microlymphatics. Lymphat. Res. Biol. 1(2), 101-119 (2003).

60. Mizuno R, Yokoyama Y, Ono N, Ikomi F, Ohhashi T. Establishment of rat lymphatic endothelial cell line. Microcirculation 10(2), 127-131 (2003).

61. Marcozzi C, Solari E, Bianchin F, Moriondo A, Negrini D. 2D and 3D cultures of lymphatic endothelial cells (LECs) from normal rat and mouse diaphragm. Acta Physiologica 200(Suppl. 681), 18 (2010).

62. Schneider CA, Rasband WS, Eliceiri KW. NIH Image to ImageJ: 25 years of image analysis. Nat. Methods 9(7), 671-675 (2012).

63. Rossi F, Bernardini G, Bonfanti P, Colombo A, Prati M, Gornati R. Effects of TCDD on spermatogenesis related factor-2 (SRF-2): gene expression in Xenopus. Toxicol. Lett. 191(2-3), 189-194 (2009).

64. Hong YK, Harvey N, Noh YH et al. Prox1 is a master control gene in the program specifying lymphatic endothelial cell fate. Dev. Dyn. 225(3), 351-357 (2002).

65. Banerji S, Ni J, Wang SX et al. LYVE-1, a new homologue of the CD44 glycoprotein, is a lymph-specific receptor for hyaluronan. J. Cell Biol. 144(4), 789-801 (1999).

66. Frattini A, Fabbri M, Valli R et al. High variability of genomic instability and gene expression profiling in different HeLa clones. Sci. Rep. 5, 1-9 (2015).

67. Vaahtomeri K, Karaman S, Mäkinen T, Alitalo K. Lymphangiogenesis guidance by paracrine and pericellular factors. Genes Dev. 31(16), 1615-1634 (2019).

68. Kilarski WW. Physiological perspective on therapies of lymphatic vessels. Adv. Wound Care 7(7), 189-208 (2018).

69. Szuba A, Skobe M, Karkkainen MJ et al. Therapeutic lymphangiogenesis with human recombinant VEGF-C. FASEB J. 16(14), 1985-1987 (2002).

70. Yoon YS, Murayama T, Gravereaux E et al. VEGF-C gene therapy augments postnatal lymphangiogenesis and ameliorates secondary lymphedema. J. Clin. Invest. 111(5), 717-725 (2003).

71. Podgrabinska S, Braun P, Velasco P, Kloos B, Pepper MS, Skobe M. Molecular characterization of lymphatic endothelial cells. Proc. Natl Acad. Sci. USA 99(25), 16069-16074 (2002).

72. Petrova TV, Mäkinen T, Mäkelä TP et al. Lymphatic endothelial reprogramming of vascular endothelial cells by the Prox-1 homeobox transcription factor. EMBO J. 21(17), 4593-4599 (2002).

73. Srinivasan RS, Escobedo N, Yang Y et al. The Prox1-Vegfr3 feedback loop maintains the identity and the number of lymphatic endothelial cell progenitors. Genes Dev. 28(19), 2175-2187 (2014).

-• Helps in understanding the feedback loop process.

74. Whitehurst B, Eversgerd C, Flister $\mathrm{M}$ et al. Molecular profile and proliferative responses of rat lymphatic endothelial cells in culture. Lymphat. Res. Biol. 4(3), 119-142 (2006).

75. Deng H, Sun C, Sun Y et al. Lipid, protein, and microRNA composition within mesenchymal stem cell-derived exosomes. Cell Reprogram. 20(3), 178-186 (2018). 